\title{
Truly Distributed Optical Fiber Sensors for Structural Health Monitoring: From the Telecommunication Optical Fiber Drawling Tower to Water Leakage Detection in Dikes and Concrete Structure Strain Monitoring
}

\author{
Jean-Marie Henault, ${ }^{1}$ Gautier Moreau, ${ }^{1}$ Sylvain Blairon, ${ }^{1}$ Jean Salin, ${ }^{1}$ \\ Jean-Robert Courivaud, ${ }^{2}$ Frédéric Taillade, ${ }^{3}$ Erick Merliot, ${ }^{3}$ Jean-Philippe Dubois, ${ }^{4}$ \\ Johan Bertrand, ${ }^{4}$ Stéphane Buschaert, ${ }^{4}$ Stefan Mayer, ${ }^{4}$ and Sylvie Delepine-Lesoille ${ }^{3,4}$ \\ ${ }^{1}$ EDF Red, 6 quai Watier, 78401 Chatou, France \\ ${ }^{2}$ EDF CIH, Savoie Technolac, 73370 Le Bourget du Lac, France \\ ${ }^{3}$ LCPC, 58 bld Lefebvre, 75015 Paris, France \\ ${ }^{4}$ Andra, 1-7 rue Jean Monnet, 92298 Chatenay-Malabry, France
}

Correspondence should be addressed to Sylvie Delepine-Lesoille, sylvie.lesoille@andra.fr

Received 1 September 2009; Revised 18 January 2010; Accepted 11 February 2010

Academic Editor: Jinying Zhu

Copyright ( $) 2010$ Jean-Marie Henault et al. This is an open access article distributed under the Creative Commons Attribution License, which permits unrestricted use, distribution, and reproduction in any medium, provided the original work is properly cited.

\begin{abstract}
Although optical fiber sensors have been developed for 30 years, there is a gap between lab experiments and field applications. This article focuses on specific methods developed to evaluate the whole sensing chain, with an emphasis on (i) commerciallyavailable optoelectronic instruments and (ii) sensing cable. A number of additional considerations for a successful pairing of these two must be taken into account for successful field applications. These considerations are further developed within this article and illustrated with practical applications of water leakage detection in dikes and concrete structures monitoring, making use of distributed temperature and strain sensing based on Rayleigh, Raman, and Brillouin scattering in optical fibers. They include an adequate choice of working wavelengths, dedicated localization processes, choices of connector type, and further include a useful selection of traditional reference sensors to be installed nearby the optical fiber sensors, as well as temperature compensation in case of strain sensing.
\end{abstract}

\section{Introduction}

Specifications for large-sized engineering structures such as the Rion-Antirion bridge (Greece) or the Millau viaduct (France) now commonly include instrumentation in order to address monitoring requirements, not only during the construction period, but also to allow lifetime structural health monitoring.

EDF's (French Electricity Company) potential applications include dams, dike and power plant reactors monitoring. Andra's (French National Radioactive Waste Management Agency) potential applications include surface and deep geological radioactive waste disposal structure monitoring, for instance, within the future geological repository that would contain highly instrumented disposal cells. LCPC is in charge of the surveillance of various French bridges developing structural pathologies due to aging.

Controlling the state of a structure's health, more commonly designated by the acronym SHM (Structural Health Monitoring), requires a large number of sensors. For this application, optical fiber sensors [1] (OFS) are found to be exceptional tools, especially as they enable distributed measurements [2] thus providing data over the entire structure instead of being limited to point data at sensor locations. Monitoring with a single fiber can thus provide information of the overall structure behavior, and 
thus overcome limitations of traditional sensors, whose information is restricted to local effects. Some 20 years of developments have been necessary to overcome the initial disappointments and fully utilize the specificities of these sensors [3], whose application has since become state of the art.

This article focuses on temperature and stain distributed sensing with optical fibers for structural health monitoring. After an initial summary description of fiber optic sensing technologies, the focus will be put on distributed temperature and strain sensors. These will be illustrated by an indepth description of two field applications: Water leakage detection in dikes using temperature distributed sensing and concrete structure monitoring by strain and temperature monitoring. Although commercially-available sensors and interrogation unit are numerous, the global measuring chain may provide disappointing monitoring results to the endusers, unless a number of specific considerations are taken into account. These are further developed within this article, with an emphasis on practical recommendations that seem essential for a successful transition from laboratory to field applications.

\section{Overview of Optical Fiber Sensor Technologies}

An optical fiber is a waveguide with a diameter in the order of $0.1 \mathrm{~mm}$ that enables conveying light upon kilometer range distances. A wide array of optical fibers, called singlemode or multimode, have been developed in either glass or plastic, with solid or hollow cores, packaged in very diverse shapes to convey signals that may be visible or invisible. As described with great details in [1], OFS offers many advantages when compared to traditional electronic sensors: they are light, small (expected to be non invasive) and insensitive to electromagnetic fields, not subject to metallic corrosion (unless a metallic cladding is chosen), and resist to high temperatures. They also make it possible to take measurements over great distances (in the kilometer range) taking advantage of very low attenuation coefficient and multiple sensors can be multiplexed in one fiber. Applications range from material sensing to oil exploration and production monitoring.

A large variety of OFS have been successfully commercialized in the past three decades essentially based on Bragg-gratings and Fabry-Perot cavities (FP), providing one or several, localized measurements [1]. These technologies require a specific, localized treatment of the fiber, for example localized surface grating, to create a localized, sensitive element susceptible to produce a measurable signal. These OFS technologies remain limited in their applications by the predefined and "point-like" nature of data they can provide. For civil engineering, a large number of such "point-like" OFS need to be multiplexed to instrument real decametric structures [4]. A wide variety of multiplexing schemes have been developed to enable simultaneous measurement of several tens of sensors. However, even if a thousand of sensors are available, the choice of their locations may be highly sensitive, and is an intensive research topic.

In comparison, distributed sensing provides a more versatile and powerful monitoring tool as it requires much less a priori knowledge of the structure behavior. The term distributed sensor designates the case in which the optical fiber itself becomes a sensor. It is thus no longer necessary to implement anticipated sensor positions since measurements are being performed all along the optical fiber connected to the reading device (as well as within the extension cables). The remainder of this article is focused on OFS technologies allowing for such distributed sensing.

\subsection{Truly Distributed Sensors: Localization Processes. Various} techniques may be utilized to develop a continuously distributed measurement system within an optical fiber. The most common would be OTDR (for Optical Time Domain Reflectometry), which could eventually be combined with a study of light-matter interactions such as the Raman effect (temperature-dependent) and the Brillouin effect (temperature and deformation-dependent) as depicted afterwards.

Initially created to analyze losses inside optical telecommunication lines [5], OTDR is categorized as an optical pulse-echo technique. The technique consists in injecting a laser pulse within an optical fiber and then measuring the backscattered intensity versus time: a period $\Delta t$ corresponds to a pulse round-trip between the lead and a given point on the fiber located at $c /(2 n \cdot \Delta t)$ from the lead. The temporal width of the pulse necessitates an OTDR spatial resolution; a 10 -ns width corresponds to a resolution of $1 \mathrm{~m}$. OTDR serves to carry out intensity variation measurements over distances in the tens of kilometers, with a spatial resolution at the meter scale.

Other localization techniques are available whose performances are quite complementary; some for example are based on frequency modulations, hence the acronym OFDR (Optical Frequency Domain Reflectometry). Detailed principle can be found in [5-7]. OFDR spatial resolution can reach $10 \mu \mathrm{m}$, although the corresponding measurement range (possible fiber length) diminishes considerably to around 100 meters.

Whatever the technique, a major issue of distributed sensing is the uncertainty on the measurement locations along the fiber as will be illustrated in the field application descriptions provided in the following.

2.2. Scattering at the Origin of Truly Distributed Sensors. As shown in Figure 1, the light backscattered by an optical fiber segment without any defects or abnormal characteristics is spectrally decomposed into three distinct peaks corresponding to three outstanding phenomena.

The first relates to Rayleigh scattering, originated from interaction between the electromagnetic wave propagating in the fiber core and silica impurities. Intensity variations in the backscattered signal at the same wavelength as the injected wave are related with local optical fiber modifications: an abrupt return peak is interpreted as a mirror reflection (connector or damage on the fiber), and a sudden drop in intensity corresponds for example to shear loss. Beyond detection, 


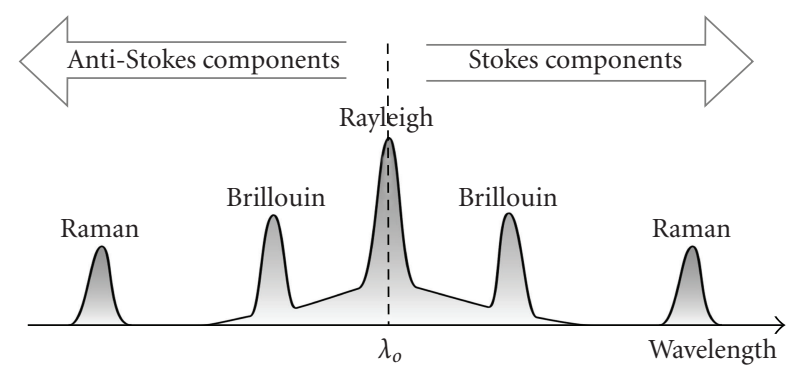

Figure 1: Backscattering spectrum of a monochromatic wave within an optical fiber.

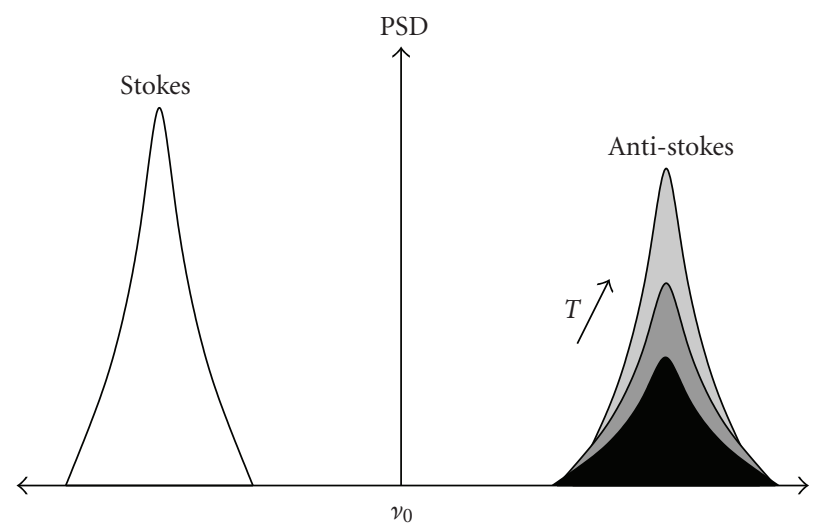

FIGURE 2: Raman scattering sensitivity to temperature ("PSD" stands for power spectral density).

to conduct temperature or strain measurements, the value of the Rayleigh backscattering signal in optical fibers must be associated with another technique, the simplest being an association with punctual sensors, such as microbend sensors or another configuration that incorporates precalibrated losses [8]. In this case, the continuously-distributed aspect of the measurement would be lost. In single-mode fibers, polarization measurements may be added [9], parameter quite interesting when application requires pressure or magnetic field sensing.

Another possibility is to perform correlation between various Rayleigh measurements as proposed by the optical backscattered reflectometry (OBR) instrument described in Section 2.2.3. An alternative is to use the two other scattering lines, Raman and Brillouin, described in Sections 2.2.1 and 2.2.2, respectively.

\subsubsection{Distributed Temperature Sensing Based on Raman Scat-} tering. To perform distributed temperature measurement, Raman scattering $[1,10]$ is the most advanced technology.

Raman scattering originates from laser light photon interaction with thermal vibration of silica molecules (thermal phonons). More precisely, as sketched in Figure 2, the anti-Stokes absorption mainly depends on temperature. As a consequence, Raman distributed sensing systems may use OTDR pulsed technique to perform distributed intensity measurement of the anti-Stokes backscattered light. However, the anti-Stokes intensity evolution must be augmented with a reference measurement since optical fiber losses vary with time (increase with fiber aging, connector dirt, or optical fiber curvatures, etc.). A number of commercially available distributed temperature sensing devices automatically compensate for this loss by analysing the ratio between the Anti-Stokes and Stokes absorption line intensities.

Paired with multimode fibers and OTDR localisation techniques, repeatability of Raman distributed temperature devices are on the order of $0.1^{\circ} \mathrm{C}$ for distance ranges of several kilometres and spatial resolution of $1 \mathrm{~m}$. It degrades with increasing distance but can be maintained by increasing the device acquisition time. Maximal distance range is $30 \mathrm{~km}$. Applications include leakage detection along oil and gas pipelines, monitoring of underground storage and boreholes, fire detection [10], monitoring of waste disposal sites and seepage detection in dams and dikes [11]. Few instruments offer the possibility to perform Raman scattering measurement into single-mode fiber. A preliminary test is reported in Section 5.2.2.

2.2.2. Distributed Temperature or Strain Sensing Based on Brillouin Scattering. As sketched in Figure 1, another inelastic phenomenon occurs when an optical pulse is launched into an optical fiber, called Brillouin scattering. This Brillouin frequency shift $v_{B}$ is linked to the acoustic mode phase velocity [12]. As a consequence, the Brillouin shift variations are known to be proportional to temperature $(\Delta T)$ and strain $(\varepsilon)$ variations [13] as in:

$$
\Delta v_{B}=C_{T} \Delta T+C_{\varepsilon} \varepsilon .
$$

$C_{T}$ and $C_{\varepsilon}$ are characteristics of the optical fiber type. At the operating wavelength $(1550 \mathrm{~nm})$, for standard G652 singlemode fiber, $C_{T}$ and $C_{\varepsilon}$ are in the order of $1 \mathrm{MHz} /{ }^{\circ} \mathrm{C}$ and $0.05 \mathrm{MHz} / \mu \varepsilon$ [13].

Instruments based on Brillouin scattering would thus perform either temperature or strain measurements. In 2002, the first commercial B-OTDR system was implemented. In 2007, the market had expanded to include at least five suppliers of Brillouin interrogation systems. The performance derived is on the order of $1^{\circ} \mathrm{C}$ or $20 \mu \varepsilon$ and $1 \mathrm{~m}$ of spatial resolution, over spans extending several tens of kilometers. The most widespread application is currently pipeline leak detection, based on temperature measurements [14]. Compared with Raman sensing, sensitivity is reduced by a factor 10 . However, Brillouin sensing enables measurement along longer distance ranges, up to $80 \mathrm{~km}$. Both differences are mainly related to the optical fiber type, respectively, multimode and single-mode for Raman and Brillouin sensing.

2.2.3. Distributed Temperature or Strain Sensing Based on Relative Rayleigh Measurements: The OBR Instrument. Another approach to perform strain or temperature distributed measurement consists in carrying out relative Rayleigh measurements, as offered by an instrument called "Optical 
Backscatterd reflectometry" (OBR) commercialized by the American company Luna Technologies.

It relies on two OFDR (see Section 2.1) measurements, the ongoing measurement and a reference state, processed with an advanced correlation method that analyses the spectral lags of the Rayleigh backscattering peak. As detailed in [7], the frequency shift $\Delta v$ of the Rayleigh backscatter pattern is proportional to temperature or strain changes along the fiber axis. For standard single-mode fiber G652 type, at $1550 \mathrm{~nm}$ typical values are $-0.1499(\mathrm{GHz} / \mathcal{E})$ and $-1.248 \mathrm{GHz} /{ }^{\circ} \mathrm{C}$.

OBR has been commercially available since spring 2006. It enables measuring approximately optical fiber deformations (at homogeneous temperature) over $100 \mathrm{~m}$ with a centimeter-sized spatial resolution and a level of precision equal to a few microstrains (resp., $0.1^{\circ} \mathrm{C}$ at homogeneous strain).

2.3. Influence of the Optical Fiber outside Coating. Up to this point, optoelectronic devices have been described. A distributed sensing system pairs such an instrument with a sensitive part including an optical fiber. A major difficulty encountered during the transition from the laboratory to the field is that the optical fiber cannot be used wrapped in its standard coatings. Either external coatings are far too fragile for outdoor test, or the coatings developed by the telecommunication industry isolate the fiber from its environment in order to protect it. The related question is how to transform an optical fiber into a transducer, as will be illustrated for the two applications described further.

\section{A Simple Qualification Methodology}

Despite many advantages described in Section 2.1, truly distributed optical fiber sensors have not invaded SHM applications yet, due to a lack of standardization in claimed performances and dedicated qualification processes.

The described overall process is inspired from [15]. The global chain evaluation must be adapted to the application. For OFS, it was chosen to study both sensing cables and optoelectronic instruments separately, before pairing such elements and focusing on data processing. Global test sequence includes the following.

(1) The SHM system is selected regarding commerciallyavailable technologies versus needs and requirements. If they do not match, internal developments are engaged, as illustrated for strain sensing cables (see Section 5.1).

(2) Special test benches are developed in laboratory to qualify metrological behaviour of the whole sensing chain under controlled conditions. For instruments, basic test can be performed in laboratory as illustrated for Raman distributed temperature device tests in Section 4.1. For embedded sensors, spatial resolution drives test structure size, which may lead to mock-up realisation as detailed in Section 4.2 for temperature sensing and Section 5.1 for strain sensing. Controlled conditions are necessary to develop quantitatively fitted data processing algorithms, as shown in Figure 7.

(3) The on field-implementation-specific requirements are finally analysed, addressed in Sections 4.3 and 5.2.3.

This qualification methodology is put in practice for two main applications: dike monitoring with Raman temperature sensing and strain distributed measurements for concrete structure monitoring. As many technical aspects were already reported elsewhere, the article rapidly mentions references dealing with the first two global test sequences, to focus on caveats for field implementation.

\section{Raman Scattering into Optical Fibers Applied to Water Leakage Detection in Dikes}

Internal erosion is a major cause of failures of embankment dams and dikes. This pathology, characterized by water leakage in the structure, remains nowadays detected by conventional method based on visual inspection. In order to improve the safety of the installation, new monitoring methods are developed.

Temperature is a good tracer of seepage [16]. Two main techniques are proposed. The passive technique is based on seasonal temperature variations in the water channel and in the ground. The active method is based on heating-up the soil.

To perform temperature measurement, optical fiber distributed sensors is a good candidate as it provides continuous monitoring in space and time. Since the beginning of the 2000s [17], EDF has launched several research programs on optical fiber instrumentation and data processing so as to get an automated Structural Health Monitoring technique able to detect seepage flows in dikes with minimized false alarm rate. The developed system relies on optical fiber technology. Although it takes advantage of commercially available elements, many difficulties had to be solved before EDF recently decided to spread the technique to various exploited structures. The following paragraphs detail difficulties to transform a laboratory instrument into a monitoring system suited to field use.

4.1. Laboratory Evaluation of Commercially Available Systems. As summarized in Section 2.2.1, several commerciallyavailable optoelectronic devices perform distributed temperature measurements using the Raman effect. As seepage detection threshold depends on the uncertainties of the measuring system, it is of major importance to determine the metrological behavior of the optoelectronic device. As direct comparison of specification sheets of the different products is not obvious, EDF decided to perform a common testing program for different commercially-available devices.

Fiber bending radius is known to influence measurement quality. Moreover, unlike standard sensors (small electronic devices), Raman measurement is averaged over 1 meter scale, because instruments rely on OTDR technique. As a result, it is mandatory to ensure a very stable temperature over 


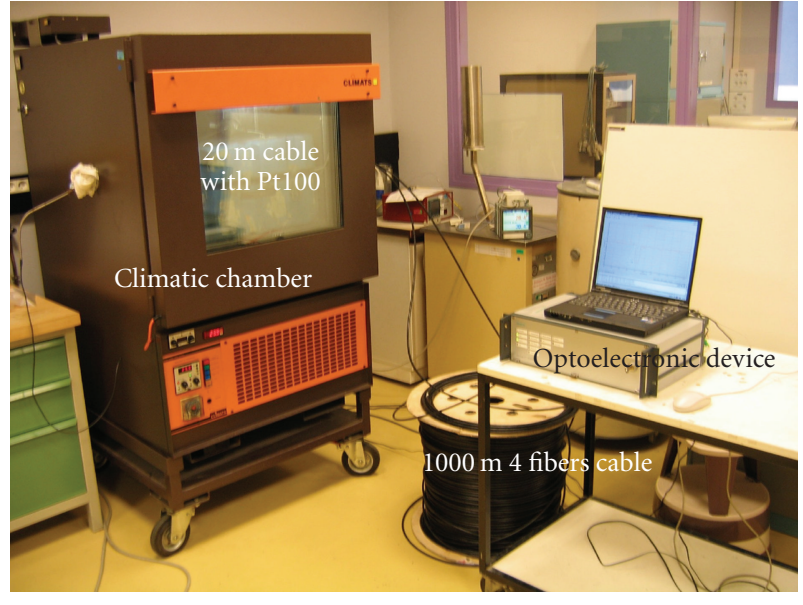

Figure 3: Different optoelectronic devices comparison thanks to a specific bench composed by a climatic chamber including a water bath equipped with fiber cables.

few meters with as less enrollment as possible. Finally, performances are expected to be closely related to average time and distance range.

A specific bench was developed, suited to water leakage application. Described in great detail in [18], it enables measuring stable temperature over $20 \mathrm{~m}$, every $1 \mathrm{~km}$, up to $4 \mathrm{~km}$. As shown in Figure 3, a commercially available cable that includes 4 multimode fibers is partially installed in a bath placed into a climatic chamber. Doing so, the cable has a minimum curvature radius of $35 \mathrm{~cm}$. Temperature is controlled with a platinum probe (Pt100 type) whose measurements show a $0.01^{\circ} \mathrm{C}$ stability over several hours. As derived from [19], repeatability is defined by the dispersion of successive measurements in the same conditions; error is derived from the difference between the device measurements and the Pt100 measurements.

Between 2005 and 2008, the same test program was applied to 7 multimode devices from different suppliers. It includes range of temperature of the bath between $0^{\circ} \mathrm{C}$ to $50^{\circ} \mathrm{C}$ and acquisition time between $30 \mathrm{~s}$ to one hour. It resulted [20] that for each device (i) the error revealed to be quite independent from the selected parameters (bath temperature, acquisition time and distance to the device) over the spans explored during the tests; (ii) the repeatability did not depend on temperature (between $0^{\circ} \mathrm{C}$ and $50^{\circ} \mathrm{C}$ ) but, as shown in Figure 4, was strongly affected by acquisition time and distance to the device.

As these laboratory tests were performed using a common procedure, the results could be compared giving a common grid evaluation that could be used to guide device choice. For instance, for an acquisition time of 5-minutes at a distance of $4000 \mathrm{~m}$ and at a temperature of $20^{\circ} \mathrm{C}$, repeatability and error of the 7 devices tests with the described procedure are given in Table 1.

If the need is to get a device which demonstrates a repeatability of $0.1^{\circ} \mathrm{C}$ and an error of $\pm 1^{\circ} \mathrm{C}$, over $4 \mathrm{~km}$ with 5 minutes acquisition time, the common comparison grid reveals that only one device is acceptable.

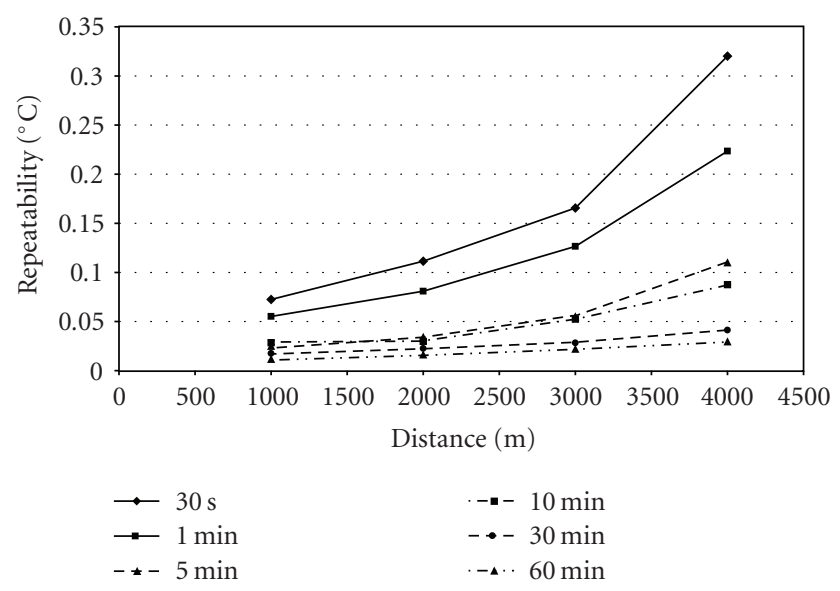

FIGURE 4: Example of repeatability results for one device tested showing the influence of acquisition time and distance from the device.

TABLE 1: Example of compared performances obtained with the described procedure for 7 devices, for an acquisition time of 5 minutes, at a distance of $4 \mathrm{~km}$ and at a temperature of $20^{\circ} \mathrm{C}$.

\begin{tabular}{lcc}
\hline Raman device no. & Repeatability ${ }^{\circ} \mathrm{C}$ & Error ${ }^{\circ} \mathrm{C}$ \\
\hline A & 0.06 & $<0.1$ \\
B & 0.11 & -0.2 \\
C & 0.11 & -0.2 \\
D & 0.29 & 4.6 \\
E & 0.43 & -0.4 \\
F & 0.57 & -0.2 \\
G & 0.58 & 0.6 \\
\hline
\end{tabular}

Meanwhile tests were being performed, Raman technology improved significantly. The comparison grid must be regularly up-dated.

4.2. Mock-up Evaluation of the SHM Technique. In order to evaluate the whole SHM system, that is to say an optoelectronic device paired with a sensing cable and a data processing method, a mock-up evaluation proved to be necessary. More precisely external parameters (air temperature, solar influx...) may compromise detection.

A full-scale moke-up was designed: a basin was built in 2006 at the Cemagref facilities, in the South of France, during the Eureka HYDRODETECT project. As described in [21] and sketched in Figure 5, the basin is composed of controlled soil materials. It enables realising artificial leaks with controlled flow rate. The optical fibers are included inside the mock-up and connected to an optoelectronic Raman device installed in a specific cabinet near the basin. Pt100 sensors, used as reference sensors, complete the instrumentation.

Raman raw measurements give a poor sensitivity to leaks (Figure 6).

As illustrated in Figure 7, specific data algorithms proved to be essential for efficient leakage detection. 


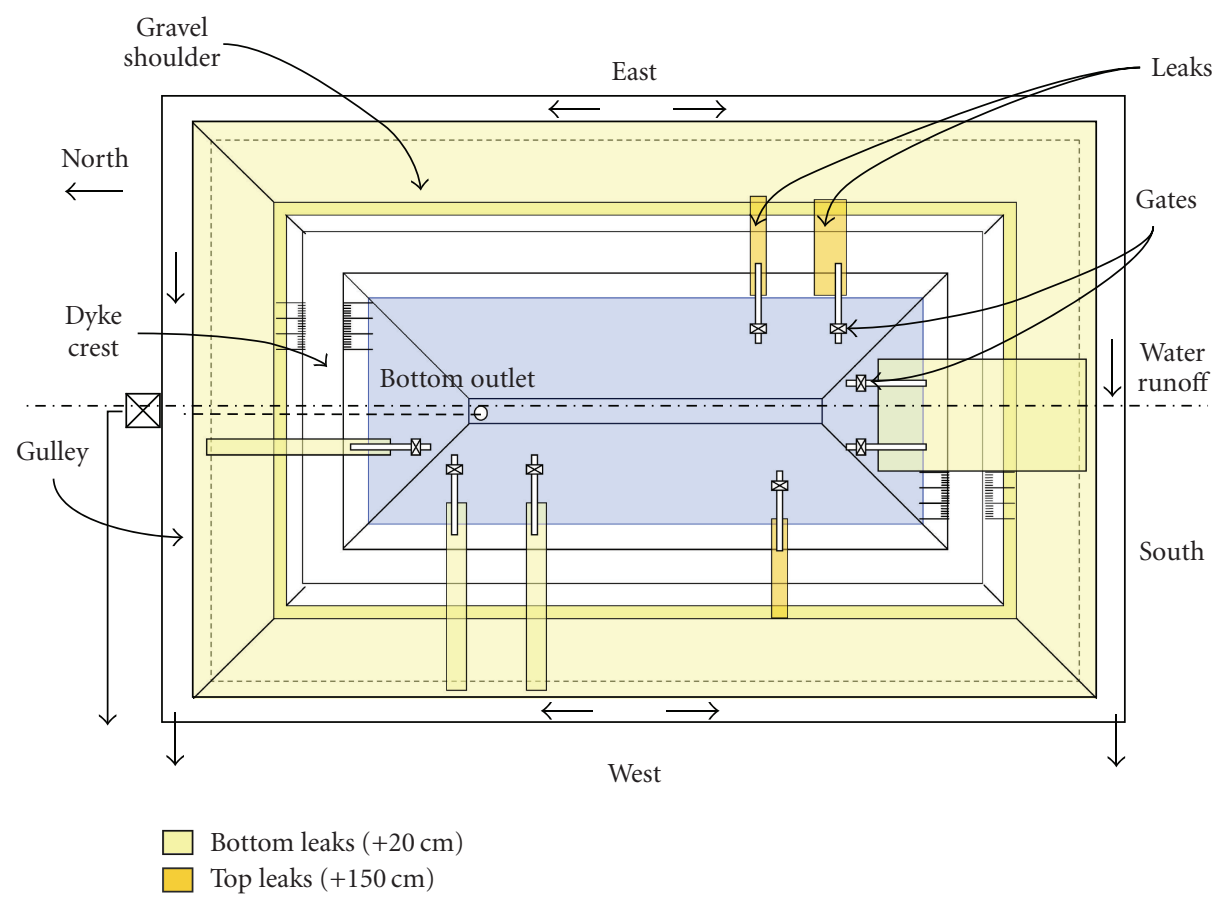

FIgURE 5: Full-scale basin equipped with 3 levels of optical fibers and artificial leaks.

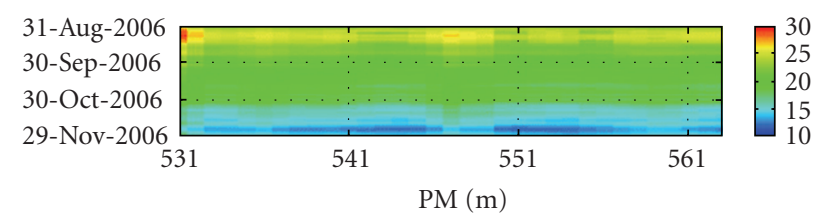

FIGURE 6: Raman temperature measurements obtained along one fiber when 3 leaks are created on the west side of the basin.

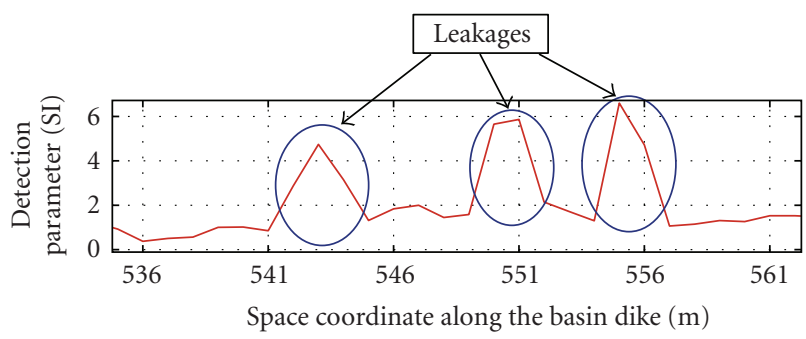

FIgURE 7: Detection of 3 leakages imposed in the West side of the basin based on raw Raman measurements given in Figure 6 processed with a specific algorithm.

At this stage, the data obtained with this mock-up enabled determining the sensitivity of the whole sensing system for leakage detection: it enables detection with a flow-rate as low as $1 \mathrm{~L} / \mathrm{m} / \mathrm{min}$ [22]. In the future, beyond detection, the data will then be used to provide quantitative information. To do so, it is planned to develop a more complex algorithm to assess leakage flow rate based on dike modelisation. The moke-up will be essential for algorithm validation as leakage flow rate cannot be accurately measured on field with traditional instrumentation as flowmeters.
4.3. On-Field Implementation. To complete laboratory and mock-up evaluations, two field installations were realized. The first dike installation is located in the South-East of France and was equipped in 2002 with a $2.3 \mathrm{~km}$ hybrid cable including 4 multimode optical fibers and 6 cupper wires. Electrical wires ensure heating mandatory to test active method [16]. Figure 8 is a picture of the dike and related cable. The second dike installation is located in the NorthEast of France and was equipped in 2006 with 2 similar cables of $1 \mathrm{~km}$. The cables are embedded at the toe of the dikes at about $1 \mathrm{~m}$ deep.

4.3.1. Material Recommendations. These installations enabled testing and validating several aspects of an on-field implementation. First, the cable has to be robust in order to endure real civil engineering works conditions: handling, soil compaction, and so forth. Moreover, it must resist to chemical aggressive environment (water and salinity). Inside dikes, rodents happened to destroy a cable, which can be solved by metallic protections. As a consequence, it is recommended to choose hybrid telecommunication cables meant for soil embedment. Moreover, its rigidity limits bending radius to approximately $0.5 \mathrm{~m}$. For instance, Leoni supplies the cable described above. 


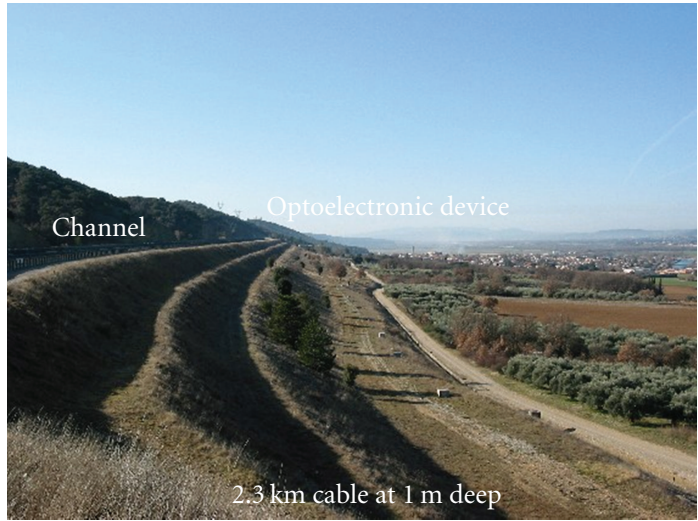

(a)

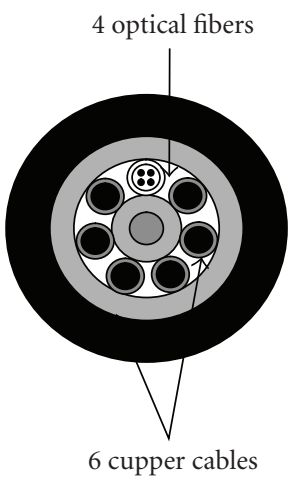

(b)

FIgURE 8: On-field installation in the South East of France.

Second, attention must be paid to selection of complementary materials. For both cases, the Raman devices were located within hydraulic power plants. The electromagnetic interferences generated by transformers and ambient temperature variations ruined measurements: approximately $50 \%$ time for the first two years of acquisition. Optoelectronic devices had to be included inside shielded and temperature regulated cabinets, with Uninterruptible Power Supply. Apart from the interrogation unit, E2000/APC connectors were chosen for eye safety reasons. At the other extremity, at the end of the cable, the fiber was placed inside a closure within a cable connection pit for ulterior extension of the installation.

While dealing with distributed data, a major difficulty is accurate event localization. Indeed, optoelectronic devices provide measurements in curvilinear abscissa along the sensing cable, which is far from the Euclidian distance at the surface of the dike. In practice, the sensing cable crosses a cable connection pit every $1 \mathrm{~km}$. Such access points enable creating artificial events by cooling or heating the cable, thus producing a clearly recognizable signal to which a known position on the structure can be attributed.

Similarly with longitudinal localization difficulty, transversal positioning is required. When an event is detected by this SHM technique, the soil needs to be dug to verify whether it is due to a leakage. To facilitate detection of cable position, in order to minimize digging works, commerciallyavailable RFID devices were buried with the cable. This significantly enhances practical use of the technique.

Third, as instrumented structures are distant from endusers, remote controlled solutions were implemented to provide rapidity in data processing and related warnings.

Last, but not least, to perform valuable measurements, Raman systems require reference measurements, as clearly learnt from experience. To do so, Pt100 are included in the cabinet to enable easy and periodic device calibration. What is more, the 4 fibers are spliced by pair at the far extremity of the cable in order to create an optical loop. As a result, the Pt100 is artificially compared to Raman measurements at two locations, one extremely closed and the second very far from the device. It also avoids maintaining reference sensors on-field.

4.3.2. Data Processing. Raman temperature measurements have been successfully acquired continuously for several years on both sites.

Various analysis methods were developed $[22,23]$ and compared. For one site, one year measurement postprocessing identified suspected areas. These results are in accordance with visual inspection reports from the owner.

4.4. Dike Monitoring Conclusions: A Success. Distributed temperature sensing thanks to Raman scattering into optical fibers was studied by EDF since the beginning of 2000s to detect leakages in dikes. The qualification methodology based on (i) laboratory (ii) mock-up evaluation under controlled conditions, and (iii) on complementary on-field tests was positively conducted. The metrological behavior of optoelectronic Raman devices of the market was evaluated. The sensitivity of the SHM technique was determined thanks to a Full-scale basin. Two industrial installations were realized in real conditions.

On the basis of the positive results obtained during the last years, EDF concludes to the qualification of this SHM technique and decides to improve the surveillance of 2 industrial sites per year by this monitoring technique. Overall results demonstrated efficiency of this whole leakage detection system based on Raman optical fiber sensing. Ongoing developments focus on quantification of leakages.

Another perspective is to take advantage of similar systems with embedded optical fibers into dikes to perform underground hole detection, as recently reported $[24,25]$. As a matter of fact, internal erosion has two main consequences: water leakage and soil deformation. For this perspective, strain distributed measurements are needed. 


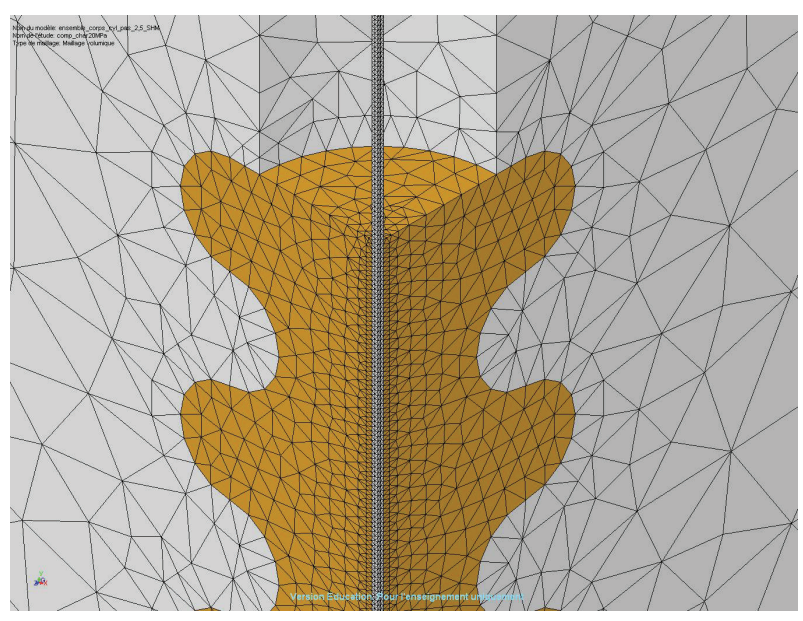

FIGURE 9: Sensor coating design with finite element calculations.

\section{Structural Health Monitoring of Concrete Structures Based on Strain Distributed Optical Fiber Sensors}

Optical fiber distributed strain sensing systems were evaluated in view of concrete structure monitoring. Instrumentation of this structure material is of major importance for Andra, as underground repository galleries and intermediate level radioactive long-lived waste disposal cells within future geological repository are likely to have an instrumented concrete lining. Similarly, LCPC and EDF are in charge of many concrete structures where safety is to be justified to authorities. Thus, monitoring is implemented in power plants, concrete dams, and a few bridges having particular features.

\subsection{Sensing Cable Design and Experimental Validation. As} mentioned in Section 2.3, special attention is to be paid to the way the optical fibers are linked with the structure, in order to perform accurate distributed temperature and strain measurements.

LCPC started developing a sensing cable dedicated to concrete instrumentation in 2002 within the EOLBUS French national project. At that time, distributed strain Brillouin sensing units were becoming commercially available, but very few related sensors were offered. More precisely, in order to perform continuous measurement of concrete strain over very long distances, the challenge is to ensure a continuous link between the host material and the optical fiber with an optimized transfer of strain and temperature fields.

As described in great detail in [26], a composite-made wave-like sensor coating was designed so as to enable continuous bonding between optical fiber and concrete. As illustrated in Figure 9, finite element analysis showed that the stiffness of the optical fiber can be adapted to the concrete's, reducing strain concentrations and the need for a theoretical calibration factor. Moreover, unlike the body of traditional Ishaped sensors (for instance vibrating wire sensor), wave-like

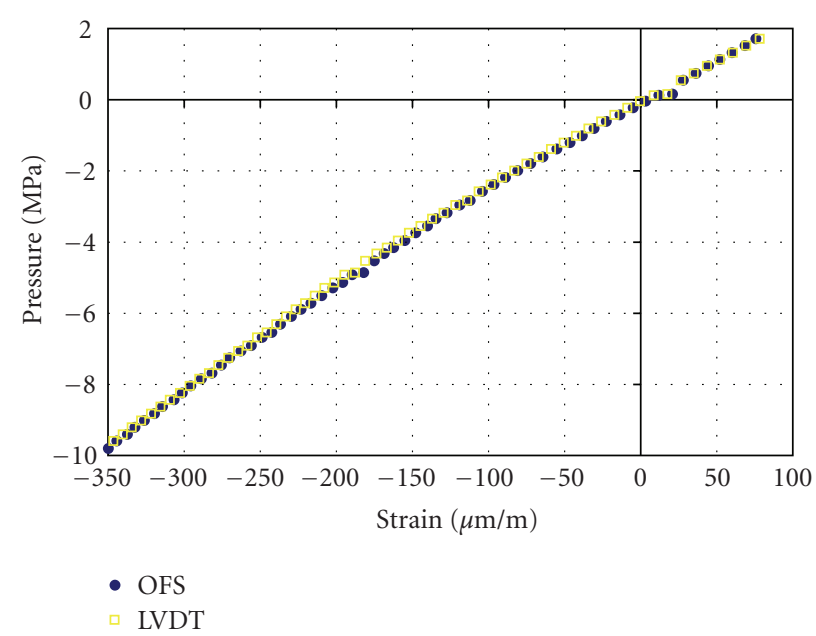

Figure 10: Experimental laboratory validation of interferometric wave-like sensors placed into small-concrete samples.

sensor body should enable symmetrical response in tensile and compressive loadings whatever the contact conditions may be.

First, experimental validations were performed with lowcoherence interferometry measurements instead of truly distributed measurements. Indeed, the $1 \mathrm{~m}$ spatial resolution of Brillouin-OTDR instruments prevents from direct comparison with reference sensors whose lengths are around $10 \mathrm{~cm}$. Partial mirrors were inserted inside the optical fiber core so as to realise short optical fiber gauges that can be interrogated by a low-coherence interferometer [26]. Experiments with $10 \mathrm{~cm}$ sensors embedded into concrete cylinders placed under press (see Figure 10) as well as field trials with $70 \mathrm{~cm}$ sensors [27] showed a very good agreement between the wrapped optical fiber extensometers and the reference extensometers placed nearby. Strain measurement threshold as low as $\pm 1 \mu \mathrm{m}$ per meter of extensometer basis was reached. Despite its wave-shape, the proposed sensor body does not introduce any losses or strain that would result from fiber microbendings.

Then, the experimental validations were performed with truly distributed measurements [28]. One major difficulty encountered at this stage was the realization of representative-scale test structures, compatible with the $1 \mathrm{~m}$ spatial resolution of commercially-available Brillouin-OTDR (at the time, Brillouin instruments with centimetric spatial resolution were restricted to laboratory experiments). A $3 \mathrm{~m}$ long concrete beam $\left(300 \times 50 \times 25 \mathrm{~cm}^{3}\right)$ was equipped with electronic temperature sensors and mechanical strain gauges near $2.8 \mathrm{~m}$ optical fiber sensing cables. These sensors were composed of standard single-mode optical fiber (G652 type and others) wrapped into the wave-like composite coating and paired with a commercially available BrillouinOTDR. Temperature measurements during concrete beam casting agreed with reference measurements, and showed the significant sensor coating influence. A month later, strain measurements performed during a four-point bending experiment showed promising results: linearity and 


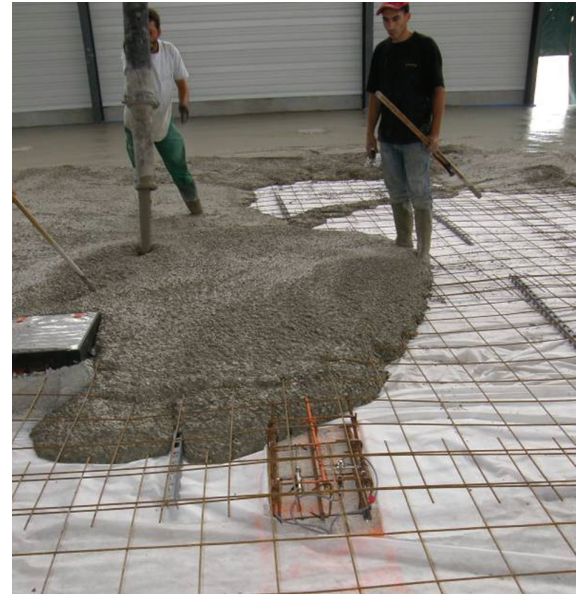

FIGURE 11: Picture of concrete slab pouring when concrete embeds reference sensors.

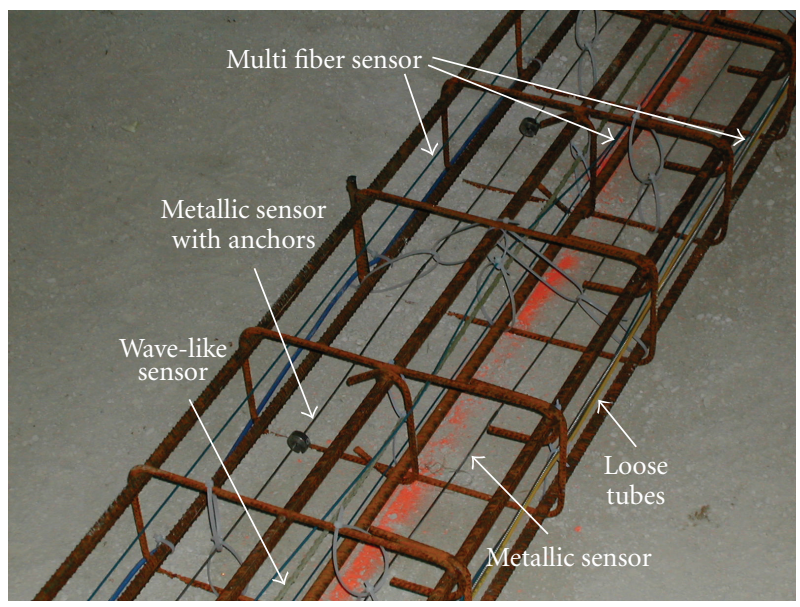

Figure 12: Optical fiber sensing cables fixed on reinforcing bar frame embedded into a concrete slab.

reliability of measurements were demonstrated, under tensile as well as compressive loadings.

These developments lasted from 2002 to 2006. They highlighted that Brillouin sensing practical implementation is restrained by three major limitations: (i) the $1 \mathrm{~m} \mathrm{spa}$ tial resolution, (ii) the poor repeatability in the order of magnitude of $1^{\circ} \mathrm{C}$ and $20 \mu \mathrm{m} / \mathrm{m}$, and (iii) the separation of temperature and strain influences.

Spatial resolution has recently been improved with centimeter spatial resolution claimed by two industrial suppliers of Brillouin instrument and extensively demonstrated in laboratories [6, 29-32]. Moreover, although not based on Brillouin scattering, the OBR instrument claims distributed strain sensing with the centimeter spatial resolution (see Section 2.2.3). Separation of temperature from strain influence has also been extensively studied for the last 3 years. Both improvements have been tested in an outdoor test described in Section 5.2.3.
5.2. Outdoor Test. As illustrated in Figure 11, Andra's Technological Exhibition Facility construction was an opportunity to compare the laboratory performances of optical fiber measuring chains (sensors and optoelectronics) with field conditions. It is a $4700 \mathrm{~m}^{2}$ industrial building hosting exhibits of various prototype equipments such as containers, large machines for radioactive waste handling (for instance, a waste disposal container pushing robot), with the purpose of communicating with the general public and enhancing their understanding of geological repositories for radioactive wastes.

As illustrated in Figure 12, several optical fiber sensing cables were installed at the core of the building concrete slab during summer 2008 construction. Five commerciallyavailable sensors meant for distributed strain sensing had been selected after prior mechanical and thermal laboratory tests. Only two appeared promising for strain distributed sensing, among which the wave-like sensor depicted in Section 5.1.

5.2.1. Material Recommendations. Sensor connections are of major importance. They necessitate extreme care during any handling which slower the instrumentation process. The optical fiber line was $250 \mathrm{~m}$ long interrupted by 18 FC/APC connectors and two splices. Unexpectedly, few fiber splices were degraded, whereas connectors appeared well suited even in terms of power budget. For civil engineering application, standard telecommunication splices revealed too stiff. Yet connectors are likely to degrade because dust size is similar with optical fiber core one.

Uncertainty on the measurement location remains a challenge. Following prescription proposed in [33], as the fiber line was composed of different sensor types, all assigned to a specific Brillouin frequency shift, localisation was already ensured every $10 \mathrm{~m}$. Moreover, inspired by dike instrumentation know-how, the instrumentation map was drawn with a heating device enabling various controls prior to concrete pouring.

5.2.2. Data Processing: Temperature Influence Compensation. To filter out temperature influence on strain measurements performed by Brillouin scattering instruments, various methods were developed based on the signal analysis [34] or the choice of the optical fiber type [35-37]. These solutions remain at a research and laboratory stage. As industrial solutions, loose tubes (where the optical fiber is supposed to be mechanically isolated) are often implemented, sometime inserted inside the same sensing cable [14]. An alternative is to pair a single-mode Raman device (single-mode is mandatory for strain sensing) with a distributed strain sensing instrument into a single optical fiber. These two industrial solutions were implemented during concrete pouring of Andra Technological Space slab: loose tube and combination of Raman and Brillouin scattering were implemented for temperature compensation.

The results reported in [38] and illustrated in Figure 13 highlighted the need for careful means while associating Raman and Brillouin technologies. Raman temperature 


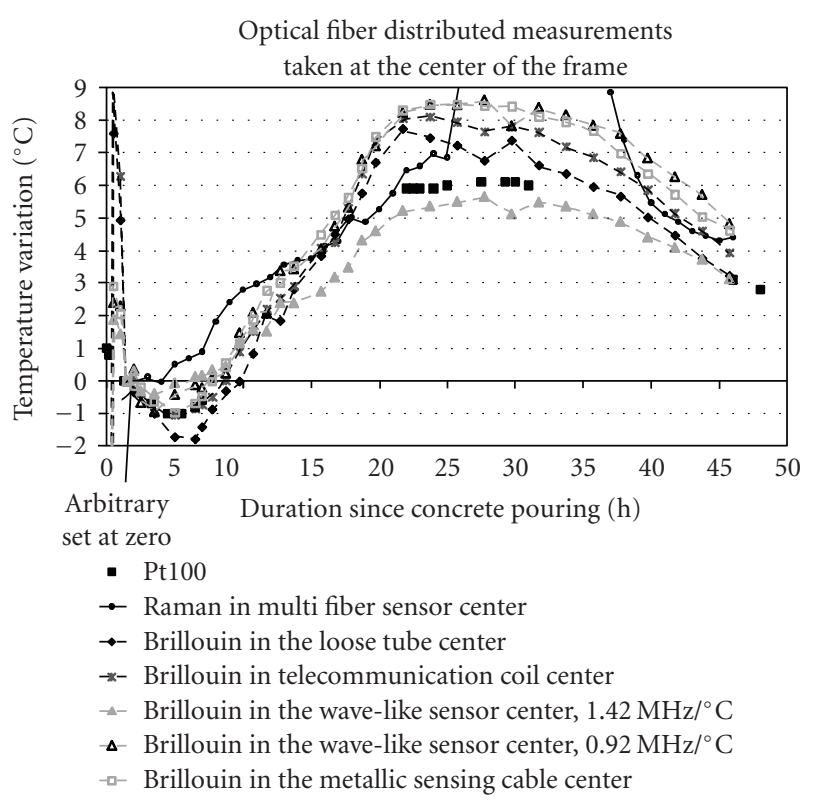

FIGURE 13: Distributed temperature measurement during concrete hardening: Reference sensors versus Raman and Brillouin distributed optical fiber sensors taken at the center of the frame.

measurements turned out to be more accurate than B-OTDR ones. The confidence acquired for dike instrumentation presented in Section 4 is, however, restricted to multimode Raman distributed temperature sensing, which was also previously tested in the laboratory. When single-mode fibers were used, sensitivity to curvature was enhanced and skewed measurements obtained during our field application (see Figure 13 after 25-hour measurements). Therefore, as no Brillouin device is available to provide measurements on multimode fibers, the necessary use of single-mode fibers prevents a combination with Raman measurements on the same fiber. While choosing a Raman single-mode device, special attention must be paid to the optoelectronic instrument working wavelength related to the sensing cable fiber. Raman Stokes intensity is a few times larger than anti-Stokes intensity and its bandwidth is usually much larger than the pump spectrum which degrades accuracy. Moreover, some of the phenomena at the origin of optical fiber losses (bending losses especially in single-mode fibers, hydrogen darkening...) are wavelength-dependent, which may ruin the method mentioned in Section 2.2.1, based on the ratio of the intensity of the two Stokes and Anti-Stokes lines: the wavelength separation between these two lines reaches $80 \mathrm{~nm}$ at $1.55 \mu \mathrm{m}$ (typical single-mode device working wavelength) versus $10 \mathrm{~nm}$ at $514.5 \mathrm{~nm}$. Recent developments implemented in most recent instruments combine various laser wavelengths [39]. To conclude, single-mode Raman device advantages and drawbacks are to be evaluated as proposed in Section 4.1.

The test of the alternative solution, the association of Brillouin instrument with sensing cable isolated form mechanical solicitation, is also detailed in [38]. It has been observed that loose tubes suffered from frictions were locally

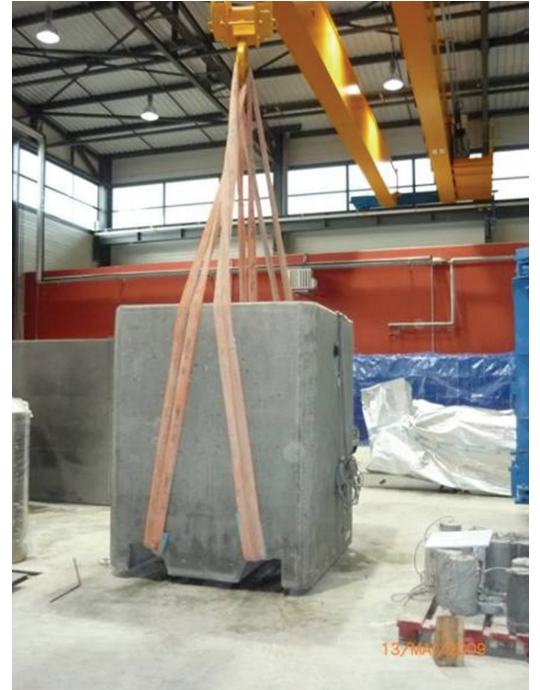

FIgURE 14: Picture of containers movements on the instrumented concrete slab.

sensitive to strain and therefore cannot be yet considered as temperature sensors while paired with B-OTDR.

\subsubsection{Strain Sensing Outdoor Test with OBR (Correlated} Rayleigh Measurements). The instrumented part of the concrete slab is now hosting heavy concrete containers, up to 15 tons that can be handled thanks to an overhead travelling crane. Distributed strain measurements can thus be performed.

Among the five sensing cables (2 loose tubes, 2 metallic sensors, 1 wave-like sensor), one broke during the first months after concrete pouring. It could be withdrawn of the sensing chain thanks to a connection pit inspired by dike instrumentation know-how, as detailed in Section 4.3. Without such care, the entire distributed measurement system would have been compromised.

Brillouin-OTDR measurements were performed few months after pouring. Seasonal thermal variations are the main detected phenomenon. Few measurement points do not follow the global sensing chain behavior, but the $1 \mathrm{~m}$ spatial resolution and the $20 \mu \mathrm{m} / \mathrm{m}$ repeatability limit accurate interpretation. New optoelectronic Brillouin units with improved spatial resolution have not been tested yet. The distributed strain sensing experiment described in this subsection deals with relative Rayleigh measurements performed by the OBR instrument.

Inside the same sensing line, measurements were performed in June 2009 with the OBR instrument described in Section 2.2.3. As relative measurements are needed to derive strain, concrete containers were (i) lifted as illustrated in Figure 14 and then (ii) piled up one on the other.

Although total weight could reach $19 \mathrm{~T}(6.6 \mathrm{~T}$ and $12.4 \mathrm{~T}$ for first and second containers, resp.), induced strain in the slab did not exceed a few microstrains. With such small events, temperature variations, although smaller than $0.8^{\circ} \mathrm{C}$, induced misinterpretations. Once corrected, the two types 


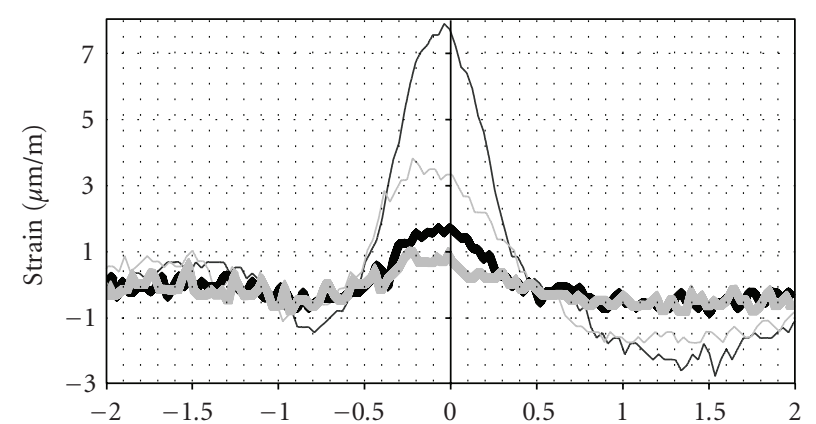

Distance along optical fiber $(\mathrm{m})$, centered on the container position

$$
\begin{aligned}
& \multimap \text { Metallic sensor-1 container } \\
& - \text { Metallic sensor-2 containers } \\
& \longrightarrow \quad \text { Wave-like sensor-1 container } \\
& \square \text { Wave-like sensor-2 containers }
\end{aligned}
$$

FIGURE 15: Distributed strain measurement performed by relative Rayleigh-OFDR measurements along two different strain sensing optical fiber cables.
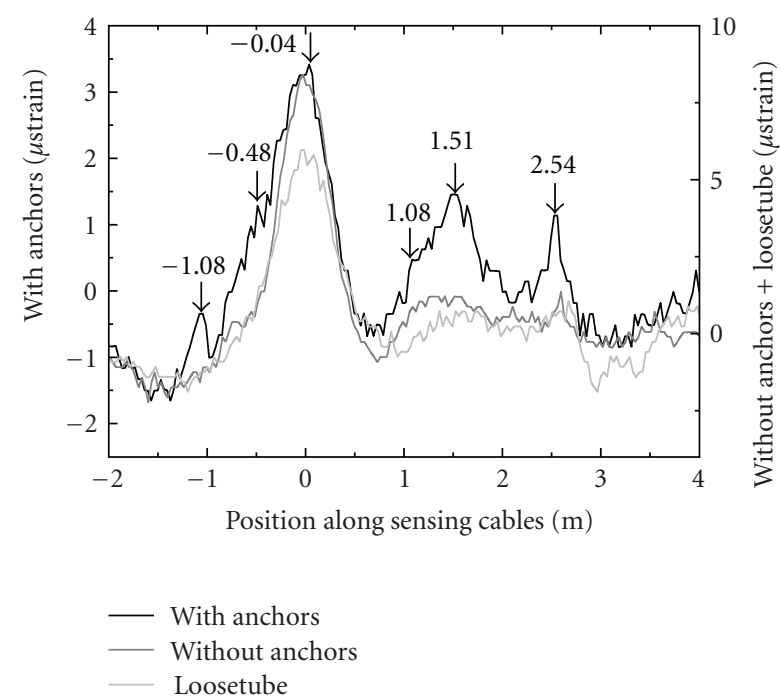

FIGURE 16: Distributed strain measurement performed by relative Rayleigh-OFDR measurements along the "loose tube" and the two metallic sensors, highlighting the influence of the anchors.

of optical fiber sensor coating, namely, wave-like composite coating and metallic coating, detect charges as showed in Figure 15. Differences between the two sensors can be correlated with (i) cable transfer functions and (ii) slightly different locations. As stated in Section 2.3, this experimental test highlights the influence of the sensor coating.

Another example of sensor coating influence is shown in Figure 16, which plots the measured strain along the loose tube and the two metallic sensing cables. As a reminder (cf. Figure 12), these metallic sensing cables differ by the addition of anchors every $50 \mathrm{~cm}$ on one of them, with the intention to enhance sensor adhesion to concrete. Localized strain peaks are observed every $50 \mathrm{~cm}$. They reflect artificial measurements induced by the presence of these anchors. The anchors' addition thus revealed unnecessary (it did not improve sensor adhesion to concrete) and degraded the strain field transfer function.

Figure 16 also showed that the specific loose tubes dedicated to temperature measurement and theoretically insensitive to strain were as sensitive to strain as the strain sensing cables. At this stage, it would however seem premature to generalize this result to all commercially available loose tubes.

Moreover, false events occurred along all sensing cables (not shown), in locations corresponding to outside coating variations, such as entrance into protective tubes to reach extracting cable room. Importance of an accurate instrumentation map is enhanced. As will be described elsewhere, measurements are also in great accordance with theoretical response and with the VWS placed nearby. This test confirmed that distributed sensing gives access to the complete data curve describing structure response to a solicitation, and thus allows for example to locate and measure the strain field maxima. Results obtained using only localized sensors are highly sensitive to the initial choice of location and may "miss" the location and magnitude of maximum strain. This outdoor test, despite the difficulties and lessons learnt in relation to adequate choice and implementation of material, as well as to adequate temperature compensation, could nevertheless demonstrate the usefulness of distributed measurements for structural health monitoring. It therefore highlights the key advantages of distributed optical fiber sensors over localized, optical fiber of other traditional sensors, in providing for a more comprehensive monitoring of a civil engineering structure.

5.3. Conclusions on Strain Sensing in Concrete Structures. As a conclusion, strain distributed sensing systems are still limited by temperature influence. Neither loose tube nor association with Raman instrument proved to be efficient within the described field applications. For concrete structure monitoring, sensing systems based on BrillouinOTDR are limited by poor accuracy ( $1 \mathrm{~m}$ spatial resolution combined with $20 \mu \mathrm{m} / \mathrm{m}$ resolution). However, using the same fibers and pairing them with a high-spatial resolution and high-strain-sensitive optoelectronic instrument (OBR), two types of strain sensing cable showed very promising results to monitor concrete slab evolutions.

\section{Overall Conclusion}

Civil engineering instrumentation is in rapid evolution, in particular because optical fiber sensors are moving from the laboratories to the field applications after almost 30 years of developments.

Various types of optoelectronic instruments are commercially available and may be paired with many different sensing cables to provide either temperature or strain distributed measurements. As a result, it may be difficult to select the combination of technologies (sensing cable and optoelectronic interrogation method) best suited for a given application. 
Two civil engineering applications were described: water leakage detection in dikes and concrete structures monitoring. Laboratory validation process revealed mandatory before any outdoor experiment. Specific methods are proposed to evaluate (i) commercially-available optoelectronic instruments namely, Raman distributed temperature instruments and (ii) sensing cable including optical fibers for strain sensing. Pairing these different sensing chain components needs advised end-user as successful outdoor tests require careful consideration of all elements influencing the measurement chain, as well as considerations related to an adequate choice of reference sensors and related to temperature compensation. A number of recommendations and lessons learnt were described in this article.

In particular, signal location along the distributed measurements over the fiber, and the correlation with actual structure position proved to be a major issue. Descriptions of various localization processes were proposed. The importance of optical fiber ends (connectors, splice, cable connection pits) was underlined. The influence on the sensing cable outside coating has also been highlighted. For water leakage detection in dikes, advanced data processing revealed mandatory. In the future, it should enable improving the ability of the optical fiber system from detection to quantification of water leakage. For concrete structures monitoring, a remaining limitation is temperature compensation on strain measurements.

Taking into account these considerations, distributed temperature and strain sensing now proves to be an efficient and unmatched tool for structural health monitoring.

\section{References}

[1] J. M. Lopez-Higuera, Handbook of Optical Fiber Sensing Technology, John Wiley \& Sons, New York, NY, USA, 2002.

[2] A. Rogers, "Distributed optical-fiber sensing," Measurement Science Technology, vol. 10, no. 8, pp. 75-99, 1999.

[3] S. Delépine-Lesoille, E. Merliot, and Y. Gautier, "Optical fiber strain sensors for use in civil engineering: state-of-the-art, industrial applications and outlook," BLPC no. 272, OctoberNovember 2008.

[4] J. M. Ko and Y. Q. Ni, "Technology developments in structural health monitoring of large-scale bridges," Engineering Structures, vol. 27, no. 12, pp. 1715-1725, 2005.

[5] J. Beller, "OTDRs and backscatter measurements," in Fiber Optic Test and Measurement, D. Derickson, Ed., chapter 11, Prentice-Hall, Upper Saddle River, NJ, USA, 1998.

[6] D. Garus, K. Krebber, F. Schliep, and T. Gogolla, "Distributed sensing technique based on Brillouin optical-fiber frequencydomain analysis," Optics Letters, vol. 21, no. 17, pp. 1402-1404, 1996.

[7] M. Froggatt and J. Moore, "High-spatial-resolution distributed strain measurement in optical fiber with Rayleigh scatter," Applied Optics, vol. 37, no. 10, 1998.

[8] K. T. Wan and C. K. Y. Leung, "Fiber optic sensor for the monitoring of mixed mode cracks in structures," Sensors and Actuators A, vol. 135, no. 2, pp. 370-380, 2007.

[9] A. J. Rogers, "Polarization optical time domain reflectometry: a new technique for the measurement of field distribution," Applied Optics, vol. 20, no. 6, pp. 1060-1074, 1981.
[10] Z. Liu and A. K. Kim, "Review of recent developments in fire detection technologies," Journal of Fire Protection Engineering, vol. 13, no. 2, pp. 129-151, 2003.

[11] S. Großwig, A. Graupner, E. Hurtig, K. Kühn, and A. Trostel, "Distributed fiber optical temperature sensing techniqueavailable tool for monitoring task," in Proceedings of the 8th International Symposium on Temperature and Thermal Measurements in Industry and Science, June 2001.

[12] G. P. Agrawal, Nonlinear Fiber Optics, Academic, New York, NY, USA, 3rd edition, 2001.

[13] M. Nikles, L. Thevenaz, and P. A. Robert, "Simple distributed fiber sensor based on Brillouin gain spectrum analysis," Optics Letters, vol. 21, no. 10, pp. 758-760, 1996.

[14] D. Inaudi and B. Glisic, "Distributed fiber-optic sensing for long-range monitoring of pipelines," in Proceedings of the $3 \mathrm{rd}$ International Conference on Structural Health Monitoring of Intelligent Infrastructure, Vancouver, Canada, 2007.

[15] Qualification guide FD CEN/TR 14748, "Non-destructive testing-methodology for qualification of non-destructive tests," 2005.

[16] O. Kappelmeyer, "The use of near surface temperature measurements for discovering anomalies due to causes at depths," Geophysical Prospecting, vol. 5, no. 3, pp. 239-258, 1957.

[17] J.-J. Fry, "Détection de fuite sur les digues par acquisition de profils de température le long d'une fiber optique," in Sécurité des Digues Fluviales et de Navigation, Congrès Français des Grands Barrages, Orléans, France, November 2004.

[18] J.-M. Henault and S. Blairon, "Exemple d'application de capteurs à fibre optique - détection de fuites dans les digues en terre par thermométrie à fiber optique," in Colloque Contrôle et Mesures Optiques pour L'Industrie (CMOI '06), Arcachon, France, 2006.

[19] ISO/IEC Guide 99, "International vocabulary of metrologybasic and general concepts and associated terms (VIM)," 2007.

[20] S. Blairon and J.-M. Henault, "Evaluation d'interrogateurs à fibre optique pour la mesure de température," in Proceedings of the 14th International Congress of Metrology, Paris, France, 2009.

[21] C. Guidoux, Y.-H. Faure, O. Artières, et al., "Measurement results on full scale field experiment using optical fibre detection methods," Wasser Wirtschaft, vol. 97, no. 10, pp. 6668, 2007.

[22] P. Cunat, Y.-L. Beck, J.-J. Fry, J.-R. Courivaud, and J.-P. Fabre, "Leakage detection based on temperature measurement with fiber optic: methods and results," in Colloque HYDRO, Lyon, France, 2009.

[23] A. A. Khan, V. Vrabie, J. I. Mars, A. Girard, and G. D’Urso, “A source separation technique for processing of thermometric data from fiber-optic DTS measurements for water leakage identification in dikes," IEEE Sensors Journal, vol. 8, no. 7, pp. 1118-1129, 2008.

[24] V. Lanticq, E. Bourgeois, P. Magnien, et al., "Soil-embedded optical fiber sensing cable interrogated by Brillouin optical time-domain reflectometry (B-OTDR) and optical frequencydomain reflectometry (OFDR) for embedded cavity detection and sinkhole warning system," Measurement Science and Technology, vol. 20, no. 3, 2009.

[25] M. Kihara, K. Hiramatsu, M. Shima, and S. Ikeda, "Distributed optical fiber strain sensor for detecting river embankment collapse," IEICE Transactions on Electronics, vol. E85-C, no. 4, pp. 952-960, 2002.

[26] S. Delépine-Lesoille, E. Merliot, C. Boulay, L. Quétel, M. Delaveau, and A. Courteville, "Quasi-distributed optical fibre extensometers for continuous embedding into concrete: 
design and realization," Smart Materials and Structures, vol. 15, no. 4, pp. 931-938, 2006.

[27] S. Delépine-Lesoille, E. Merliot, Y. Gautier, L. Quétel, M. Delaveau, and A. Courteville, "Multiplexed long-base flexible optical fiber extensometers and temperature bragg sensors interrogated by low-coherence interferometry," IEEE Sensors Journal, vol. 8, no. 7, pp. 1145-1151, 2008.

[28] V. Lanticq, M. Quiertant, E. Merliot, and S. Delépine-Lesoille, "Brillouin sensing cable: design and experimental validation," IEEE Sensors Journal, vol. 8, no. 7, pp. 1194-1201, 2008.

[29] L. Zou, X. Bao, Y. Wan, and L. Chen, "Coherent probe-pumpbased Brillouin sensor for centimeter-crack detection," Optics Letters, vol. 30, no. 4, pp. 370-372, 2005.

[30] K. Hotate and T. Hasegawa, "Measurement of Brillouin gain spectrum distribution along an optical fiber using a correlation-based technique-proposal, experiment and simulation," IEICE Transactions on Electronics, vol. E83-C, no. 3, pp. 405-411, 2000.

[31] A. W. Brown, B. G. Colpitts, and K. Brown, "Dark-pulse Brillouin optical time-domain sensor with $20-\mathrm{mm}$ spatial resolution," Journal of Lightwave Technology, vol. 25, no. 1, pp. 381-386, 2007.

[32] L. Thévenaz and S. Foaleng Mafang, "Distributed fiber sensing using Brillouin echoes," in 19th International Conference on Optical Fibre Sensors, vol. 7004 of Proceedings of SPIE, Perth, Western Australia, April 2008, 70043N.

[33] N. Honda, M. Inoue, N. Araki, and Y. Azuma, "New optical fiber line testing system function for highly accurate facility location using Brillouin frequency shift assigned optical fiber," in Proceedings of the Optical Fiber Communication Conference, Optical Society of America, 2009, paper no. OWU3.

[34] P. C. Wait and T. P. Newson, "Landau Placzek ratio applied to distributed fibre sensing," Optics Communications, vol. 122, no. 4-6, pp. 141-146, 1996.

[35] C. C. Lee, P. W. Chiang, and S. Chi, "Utilization of a dispersion-shifted fiber for simultaneous measurement of distributed strain and temperature through Brillouin frequency shift," IEEE Photonics Technology Letters, vol. 13, no. 10, pp. 1094-1096, 2001.

[36] L. Zou, X. Bao, V. Afshar, and L. Chen, "Dependence of the Brillouin frequency shift on strain and temperature in a photonic crystal fiber," Optics Letters, vol. 29, no. 13, pp. 14851487, 2004.

[37] M. Froggatt, D. Gifford, S. Kreger, M. Wolfe, and B. Soller, "Distributed strain and temperature discrimination in unaltered polarization maintaining fiber," in Proceedings of the 18th Optical Fiber Sensors Conference, Optical Society of America, Cancun, Mexico, 2006, paper no. ThC5.

[38] J.-P. Dubois, S. Delépine-Lesoille, V.-H. Tran, et al., "Raman versus Brillouin optical fiber distributed temperature sensing: an outdoor comparison," in Proceedings of the 4th International Conference on Structural Health Monitoring on Intelligent Infrastructure (SHMII '09), Zurich, Switzerland, 2009.

[39] C. Lee, K. Suh, and T. Landry, "The implementation of self calibration techniques in Raman backscatter based fiber optic distributed temperature system (DTS) technology," in Proceedings of the Transmission and Distribution Conference and Exposition, pp. 1-6, 2008. 

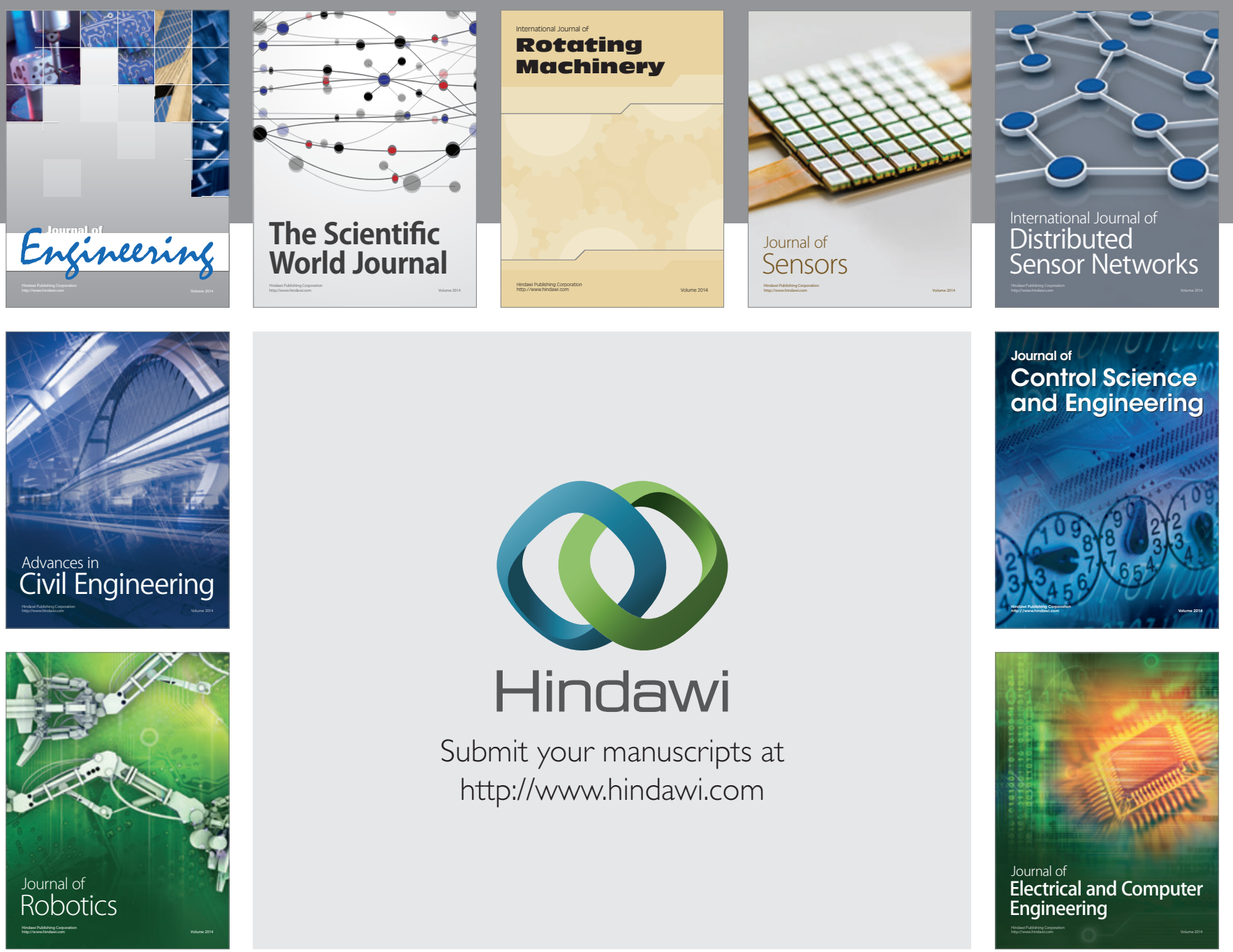

Submit your manuscripts at

http://www.hindawi.com
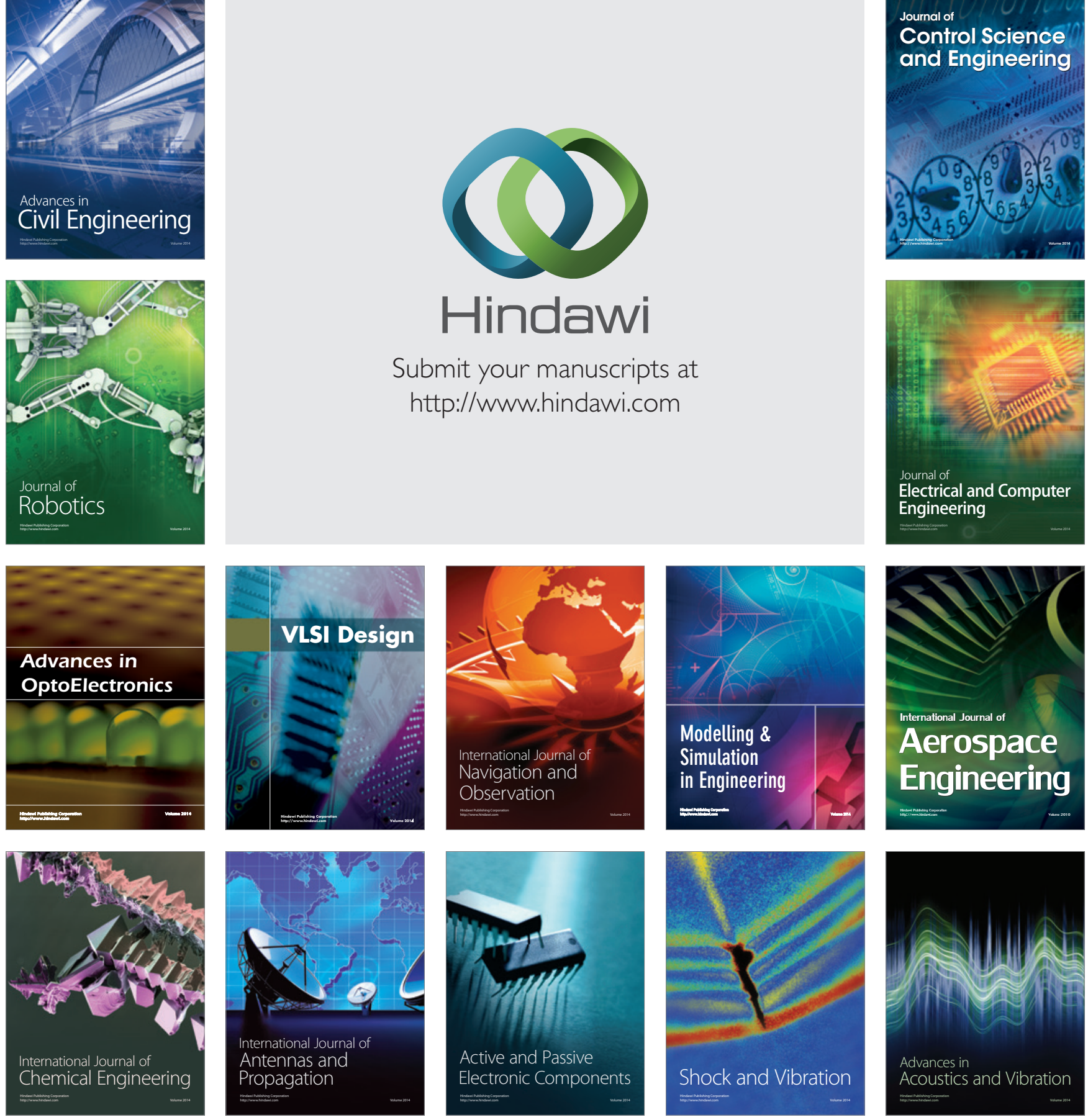